\title{
Experimentation with MANETs of Smartphones
}

\author{
Eduardo Soares*, Pedro Brandão*, Rui Prior*, Ana Aguiar ${ }^{\dagger}$ \\ *Instituto de Telecomunicações and Faculdade de Ciências da Universidade do Porto \\ \{esoares, pbrandao,rprior\}@dcc.fc.up.pt \\ †Instituto de Telecomunicações and Faculdade de Engenharia da Universidade do Porto \\ anaa@fe.up.pt
}

\begin{abstract}
Mobile AdHoc NETworks (MANETs) have been identified as a key emerging technology for scenarios in which IEEE 802.11 or cellular communications are either infeasible, inefficient, or cost-ineffective. Smartphones are the most adequate network nodes in many of these scenarios, but it is not straightforward to build a network with them. We extensively survey existing possibilities to build applications on top of ad-hoc smartphone networks for experimentation purposes, and introduce a taxonomy to classify them. We present AdHocDroid, an Android package that creates an IP-level MANET of (rooted) Android smartphones, and make it publicly available to the community. AdHocDroid supports standard TCP/IP applications, providing real smartphone IEEE 802.11 MANET and the capability to easily change the routing protocol. We tested our framework on several smartphones and a laptop. We validate the MANET running off-the-shelf applications, and reporting on experimental performance evaluation, including network metrics and battery discharge rate.
\end{abstract}

Keywords-Ad-hoc networks, MANET, mesh networks, Android

\section{INTRODUCTION}

Although Internet connectivity is nearly ubiquitous, there are many situations in which using infrastructureless communication is better than an IEEE 802.11 hotspot or cellular communication, because the latter are either infeasible, inefficient, or cost ineffective. For example, in remote areas, e.g. forests, ocean, or in catastrophe scenarios [1], [2], there is simply no infrastructure to provide connectivity. Or in social upraise scenarios, in which the infrastructure cannot be trusted, as the use of Open Garden ${ }^{1}$ shows. A more leisurely example is low latency gaming [3], [4] or sharing a file with acquaintances. Another application scenario could be group communication in mass events, like conferences or concerts [5], in which infrastructure may be unable to support all communication demand. For all these reasons, wireless ad-hoc networking was identified as a major emerging technology at the "Internet on the Move" workshop [6] and by Conti et al. [7].

Although smartphones are privileged network nodes in the people centric scenarios mentioned above, work in this direction lies primarily in the field of middleware for distributed applications, whereby connectivity is blindly assumed up to few exceptions like Haggle [8], and only a few provide support for smartphones. Recently, application frameworks that leverage WiFi Direct or cooperation between IEEE 802.11 and Bluetooth (BT) appeared. But those solutions do not create an IP-level network, require overlay routing for multi-hop communication, and applications need to be adapted to a specific framework

\footnotetext{
${ }^{1}$ http://opengarden.com/about
}

Application Programming Interfaces (APIs). IP-level multi-hop networking makes the difference in scenarios where latency is critical, and where communication with other IP enabled devices like laptops or PCs is wanted. Moreover, it is completely transparent to applications, which just use the sockets API.

In this paper, we review work on MANETs of smartphones, and proceed to extensively survey solutions that claim to provide ad-hoc connectivity for smartphones (section II) We then introduce AdHocDroid to turn smartphones into nodes of an IP-level mobile ad-hoc network. A MANET of Android smartphones enables simplified instantiation of a test-bed for experimental evaluation of routing protocols, data dissemination, distributed applications, etc. [9]. We describe how to set up an IP-level 802.11 ad-hoc network with multi-hop capability on smartphones running the Android Operating System (OS), and share lessons learned (section III). Our purpose is to advance MANET experimentation by enabling easy testing on MANET network protocols. Then, we introduce a taxonomy of MANET features and use it to characterize the surveyed solutions and AdHocDroid (section IV). Finally, we experimentally validate AdHocDroid running applications on the network, and evaluating network performance (throughput and latency) and battery consumption (section $\mathrm{V}$ ).

\section{RELATED WORK}

A MANET is a wireless ad-hoc network that allows, and adapts to, mobility of the participating nodes, which are terminals that also route packets of flows in which they are not endpoints. An ad-hoc network is any type of selfconfiguring network that does not require pre-existing installed and configured infrastructure. As such, nodes in a MANET are able to communicate with every node in the MANET as long as a path between the nodes may be established.

SocialMesh explores the idea of an ad-hoc network of citizen's devices as an infrastructure-less communication network, and its vulnerabilities to a wide range of attacks [2]. Although smartphones are identified as straightforward instantiations of nodes, the article focuses on the security aspects and evaluates them qualitatively. Fully distributed services on top of ad-hoc networks have been explored in a plethora of works, focusing on middleware that provides service discovery [1], multicast group communication [10], [3], data dissemination [5], improved latency [4], or all of them and more, like Haggle [8]. These works assume that multi-hop connectivity is provided either through IP or as overlay on IEEE 802.11 and/or BT. In this sense, this paper addresses alternatives to provide the necessary underlying multi-hop connectivity at the network layer using smartphones. 
A survey of experimental work with MANETs [9] shows that real-world experiments can bring to light significantly different behaviours than MANETs simulation or even emulation. The survey describes 5 static experiments and 8 with mobility, but only 2 testbeds (APE and ORBIT), which use laptops running the Linux OS with 802.11 dongles. The survey highlights the importance of real world-experimentation and describes toolsets. Recently, Papadopoulos et al. [11] highlighted the benefits of experimentation for the deployment of ad-hoc networks, and identify reproducibility as a caveat of the methodology. In this sense, we expect to contribute to the acceleration of experimentation with ad-hoc networks of smartphones by providing a tool that simplifies setting up such a network.

The next sub-sections describe protocols and frameworks that aim at providing MANETs.

\section{A. 802.11 Support}

The latest revision of the IEEE 802.11 standard [12] supports two different modes that can be used for ad-hoc networking: Independent Basic Service Set (IBSS) mode and 802.11 s

1) IBSS Mode: The IBSS mode of 802.11, commonly referred to as ad-hoc mode because it does not require any infrastructure to be in place, can be used as a basis for mesh networking. In this mode, all nodes play similar roles, and any node can communicate directly with any other node within the network, defined as the set of nodes in IBSS mode sharing the same Service Set Identifier (SSID) that are within its radio range.

The IBSS mode itself, however, does not provide multihop capabilities. There is no provision for path discovery and selection, nor for relaying frames to nodes out of the radio range of the original sender. In IBSS-based ad-hoc networks, these functions must be performed by an additional protocol, like OLSR [13] or BATMAN ${ }^{2}$, usually at the network layer. Since connectivity is provided at the link layer (optionally with additional network layer support for multi-hop), IP-based applications work without any modification in an IBSS-based network. Interoperation with non-Android systems works out-ofthe-box for the single-hop case, and requires a routing protocol for multi-hop.

2) $802.11 \mathrm{~s}:$ More recently, mesh networking support has been introduced in 802.11 through the $802.11 \mathrm{~s}$ amendment, now incorporated in the standard [12]. 802.11s defines the Mesh Basic Service Set (MBSS) that provides a wireless Distribution System (DS), either independent or extending the wired DS, based on meshing at the link layer. 802.11s defines a standard path metric and path selection protocol, Hybrid Wireless Mesh Protocol (HWMP), though others can be used as long as all stations in the mesh agree. HWMP combines reactive routing derived from Ad hoc On-demand Distance Vector (AODV) [14] with root-based proactive routing for communication with the outside.

In Linux, 802.11s is supported through the open802.11s implementation $^{3}$. Open802.11s supports wireless chipsets with

\footnotetext{
${ }^{2}$ https://www.open-mesh.org/projects/open-mesh/wiki

${ }^{3}$ http://open80211s.org/open80211s/
}

a driver using the software implementation of the MAC layer provided by the mac 80211 kernel module. Currently, most wireless chipset drivers use a hardware implementation of the MAC layer, and are, thus, unsupported. The driver must also be mesh-enabled. $802.11 \mathrm{~s}$ is now merged in the mainstream kernel, but support for it must be configured, and an updated version of the iw tool is also required.

In Android, vendor-provided system images do not usually support $802.11 \mathrm{~s}$ in the kernel or the configuration tools. This means that the use of $802.11 \mathrm{~s}$, even on devices with supported chipsets, is limited to those using third party, customized Android versions, and we are not aware of any that currently supports 802.11s.

\section{B. WiFi Direct}

WiFi Direct is a technical specification [15] of the WiFi Alliance that leverages existing standards to provide a convenient way for securely connecting devices without installed infrastructure, enhanced with features like peer and service discovery. It is based on the infrastructure BSS mode of 802.11. One of the devices, selected through negotiation, will become the group owner (Group Owner (GO)) and act as an Access Point (AP). This has the advantage of allowing legacy clients to connect to the GO. The GO incorporates a Dynamic Host Configuration Protocol (DHCP) server for providing IP addresses to the Client nodes.

WiFi Direct imposes a star topology, with the GO at the center. While the specification mentions Concurrent Devices that can simultaneously connect to the infrastructure or be part of a different group (requiring these devices to support multiple MAC entities), additional protocols are required for routing. A significant disadvantage of WiFi Direct is that if the GO leaves, the group is torn down and a new group must be established from scratch. While these limitations are irrelevant in simple situations like a printer letting computers and other devices connect, they make WiFi Direct unsuitable as a basis for multi-hop networking.

To the best of our knowledge, the experiments to provide multi-hop support using WiFi Direct are limited to Content Centric Network $(\mathrm{CCN})$ approaches where the routing is hidden using the search for the content. Jung et al. [16] try to use the concurrent operation mode to have the card connected to more than one point, but this work is only tested through NS-3 simulations. Content-centric device-to-device routing has been tried in non-rooted Android smartphones using WiFi Direct [17]. However, WiFi Direct in Android assigns the same IP address (192.168.49.1) to the GO of all groups. The authors proxy the connection through several nodes to circumvent this problem. These issues reveal the inadequacy of using WiFi Direct for multi-hop networks, particularly in Android.

\section{Open Garden}

Open Gardenis a software for Internet connection sharing on mobile devices using a mesh of BT or WiFi Direct links. It also allows communication between devices across multiple hops as long as the application uses OpenGarden's proprietary forwarding software. The FireChat application, from the same company, runs on top of Open Garden enabling a multihop messaging framework. From the scarce documentation 
and our tests using the software, we concluded that no IPlevel connectivity that might be used by other applications is provided.

Open Garden works by creating a Virtual Private Network (VPN) to a BT paired device also running the application. The other device terminates the VPN tunnel and either forwards the request to another node or redirects the message to the local application that registered for it (most commonly FireChat $)^{4}$. With this architecture, a multi-hop overlay network is established using BT connections.

When a device has Internet connection (through cellular or 802.11 infrastructure) it can forward the requests received. This, again, after the Open Garden software interprets and re-routes the data packets. Thus, Open Garden is also not an alternative to set up a MANET test-bed of smartphones.

\section{Serval Project}

The Serval project [18] provides a free and open-source software to allow mobile phones to communicate in the absence of phone towers and other infrastructure, targetting disaster situations and remote communities. The Serval Mesh application ${ }^{5}$ provides voice calls, text messaging and file sharing directly over IEEE 802.11 links between mobile devices. It can be used for peer-to-peer communication through an 802.11 AP or in an ad-hoc multi-hop topology without infrastructure support. The MANET is implemented using an ad-hoc routing protocol over 802.11 in IBSS mode. The project initially used BATMAN, but moved to an in-house routing protocol.

The project developed Mesh Datagram Protocol (MDP), a hybrid of network and transport layer protocol that shares some properties with User Datagram Protocol (UDP), but with per-hop retransmission of packets for mitigating the cumulative end-to-end packet loss effect that can significantly affect the performance of multi-hop wireless environments. MDP can work over IP, or directly over link layer technologies ${ }^{6}$. On top of MDP, the project provides Rhizome, a resilient file distribution protocol that is used to transparently transport data across the mesh nodes. It is used for transmitting messages or support other services, such as their Voice Over Mesh Protocol (VoMP). The project also defines a Distributed Numbering Architecture (DNA) to identify and address the nodes with cryptographic IDs on the network.

The current application on the Google Play Store includes the Serval Mesh that provides the above functionality including the project's routing protocol. Currently the development is being driven for mobile phones and Android is the one currently supported with applications. The specificities of the protocols outlined above make the Serval approach unusable by applications that are not aware of their API and sub-system. This provides little to no flexibility as a MANET test-bed.

\footnotetext{
${ }^{4}$ We were unable to verify the level of node identification used. From our experiments, it seemed that this was carried in a proprietary message that the terminating point (Open Garden software) would interpret. One indication of this is that the IP address of the VPN tunnels were the same on all devices.

${ }^{5} \mathrm{http}: / /$ developer.servalproject.org/dokuwiki/

${ }^{6}$ The intention of supporting MDP over BT is stated.
}

\section{ADHOCDROID}

AdHocDroid is an Android application that makes the necessary changes in the device to effortlessly create a MANET in one step, as shown in Fig. 1. The application sets up the IBSS network, enabling ad-hoc mode on the wireless card, offers the possibility to choose the network name, and configures the IP address, network mask and gateway for the device. All parameters have default values, e.g. the IP address is chosen according to [19]. The application also allows an easy way to import and run different routing protocols, and using tools to monitor and evaluate the state of the network.

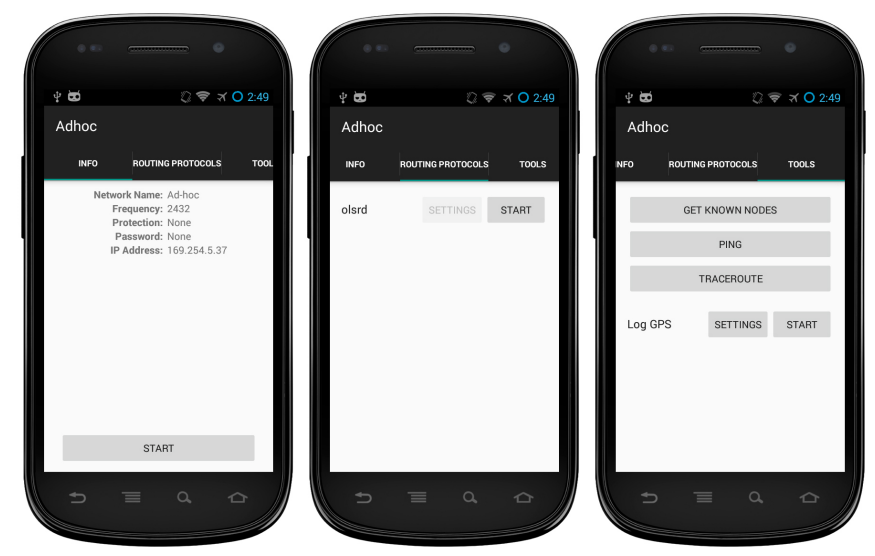

Figure 1. AdHocDroid application screen-shots

We where able to successfully use our application enabling multi-hop connectivity on the Gigabyte Gsmart G1305 with Android 2.3 (CyanogenMod 7), and Samsung Nexus S with Android 4.3 (CyanogenMod 10.2.1) smartphones, and on the Samsung Galaxy Tab 10.1 tablet with Android 3.2. We also tested it on LG Nexus 4, LG Nexus 5 and Motorola Moto G (2013), but due to driver or chipset issues the AdHoc mode did not function correctly. We have also tested the smartphone ad-hoc network with a 1 st responder monitoring application with success [20].

\section{A. Architecture}

The application follows a modular architecture as shown in Fig. 2. There are three clearly defined modules that map to the application seen in Fig. 1 (left-to-right), the network configuration (NetConfig), the routing protocols (Routing) and tools (Tools).
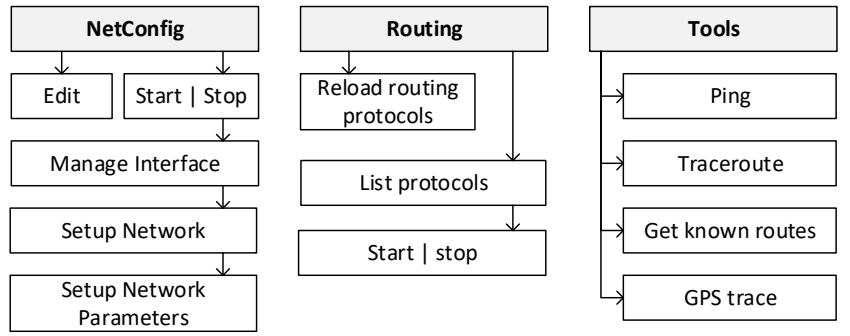

Figure 2. AdHocDroid application architecture

For the network configuration all parameters can be adjusted. The start/stop button executes the network stack setup procedure. 
First it turns off the network interface via the Android API, which avoids any other application (third party or even the system settings) making changes to the configurations of the networks. It then alters the text file that stores all known networks to add or remove the IBSS network (the wpa_supplicant.conf). It finishes turning on the network interface in order to load the new network, and apply to it the network parameters (IP address, network mask, gateway).This last step issues commands on the command line.

For multi-hop connectivity, we bundle the application with an $\mathrm{OLSR}^{7}$ routing daemon, but it is easy to import, start and stop other routing protocols. This can be done without recompiling or changing AdHocDroid, by creating a zip file with bash scripts for starting and stopping the routing protocol, and the executable binaries with the implementation of the protocol cross-compiled to the architecture of the device. We provide more detailed documentation on how to add and use the pre-compiled OLSR build that runs on Android devices as an example in https:/github.com/eSoares/ Routing-Protocol-package-to-Android-Ad-hoc-framework. Using this routing protocol, we verified that it is possible for terminals running other OS's to join the created MANET. This was tested with a laptop running a Linux distribution (Ubuntu) with OLSR and two smartphones compatible with AdHocDroid.

AdHocDroid has additional tools that we have found useful when carrying out experiments in the field, like providing information on the routing table, and execute ping or traceroute commands. It is also possible to log the smartphone GPS coordinates, e.g. to map connectivity using geographic positions.

\section{B. Lessons Learned}

During development and testing, we came across problems with the Android APIs and the diversity of devices, which we report in this section.

The Android API to configure network information, namely IP address, gateway and network mask, was deprecated in Android HoneyComb (3.0). To surpass this problem we used reflection through undocumented and internal APIs, and wrapped this in a library, which we published in https: //github.com/eSoares/Android-IP-Manager.

The Android API does not enable the creation of an adhoc network. Thus, the library directly edits, as described, the system configuration file, usually wpa_supplicant.conf in Linux distributions, located in /data/misc/wifi/ in Android. However, we found that it has other names in some devices. For example, in Samsung Galaxy Tab 10.1 it was named bcm_supp.conf and was located in /data/wifi/.

Sometimes, after adding a new IBSS network, the phone would prefer a previously saved 802.11 network instead of connecting to the new network. To fix this, the library edits wpa_supplicant.conf to show only IBSS networks to the OS when we want to connect to ad-hoc networks.

Even after the network was completely configured and set up, some devices (LG Nexus 5 and Motorola Moto G (2013)) were unable to connect to the MANET. We assume that the WiFi chipset drivers did not implement this mode, since the

\footnotetext{
${ }^{7}$ See http://www.olsr.org/
}

Android OS did not present any limitation and messages of issues in the driver where present in the Android WiFi state machine.

Some other devices (LG Nexus 4) are able to connect to the network in IBSS mode, but we found non-compliant behaviour. The first device to connect would act as an AP, and other devices would from then on use this AP to route traffic. If the first device (acting as AP) left or went out of range, the rest of devices in the network where unable to communicate.

In summary, IBSS mode in Android devices is problematic mainly due to drivers or chipsets not implementing the required functionality. Nevertheless, we tested and were/are able to run AdHocDroid repeatedly and consistently on Gigabyte Gsmart G1305, Samsung Nexus S and Samsung Galaxy Tab 10.1.

\section{Is It REALly A WiRELESS MANET?}

Given that some applications claim to provide mobile ad-hoc networking, we set off to think about what defines a MANET. In our view, that definition requires answering all the following questions with yes:

- is communication possible without connectivity to the Internet? (No Internet Needed)

- is multi-hop communication possible? (Multi-hop)

- can any application take advantage of the provided connectivity through a regular socket API, thus not requiring adaptation/re-writing? (Any App)

- can work without needing additional wireless technology to provide communication, e.g.: using IEEE 802.11 needs also BT? (No other Wireless)

- can we use off-the-shelf OS's to communicate with the MANET? E.g.: if development is on Android can we communicate with a PC running another OS? (Other Systems)

We analysed the technologies addressed in section II according to this definition, and summarise the results in table I.

The main problem that almost every proposal faces is the support in different systems. This in some cases may be a matter of adoption (802.11s and AdHocDroid) while in others it involves "heavier" development from the proponents themselves. Some points are critical for a MANET testbed namely supporting multi-hop and providing the regular socket interface for applications. In our view, this makes Open Garden, Serval and WiFi Direct definitively not fit the "wireless MANET" name.

Table I. MANET NETWORK SOLUTIONS CHECK-LIST. yes IS DENOTED $\mathrm{BY} \checkmark$, no $\mathrm{BY} \times \mathrm{AND} \bullet \mathrm{INDICATES}$ partially $\mathrm{OR}$ with some adaptations

\begin{tabular}{|c|c|c|c|c|c|}
\hline Proposal & $\begin{array}{c}\text { No } \\
\text { Internet } \\
\text { Needed }\end{array}$ & $\begin{array}{c}\text { Multi- } \\
\text { hop }\end{array}$ & $\begin{array}{c}\text { Any } \\
\text { App }\end{array}$ & $\begin{array}{c}\text { No other } \\
\text { Wireless }\end{array}$ & $\begin{array}{c}\text { Other } \\
\text { Systems }\end{array}$ \\
\hline $\begin{array}{c}802.11 \text { s } \\
\text { (native) }\end{array}$ & $\checkmark$ & $\checkmark$ & $\checkmark$ & $\checkmark$ & \\
\hline $\begin{array}{c}\text { Open } \\
\text { Garden }\end{array}$ & $\checkmark$ & $\checkmark$ & $\times$ & $\times$ & $\times$ \\
\hline Serval & $\checkmark$ & $\checkmark$ & $\times$ & $\checkmark$ & $\times$ \\
\hline $\begin{array}{c}\text { WiFi } \\
\text { Direct }\end{array}$ & $\checkmark$ & $\times$ & $\times$ & $\checkmark$ & $\checkmark$ \\
\hline $\begin{array}{c}\text { AdHoc- } \\
\text { Droid }\end{array}$ & $\checkmark$ & $\checkmark$ & $\checkmark$ & $\checkmark$ & \\
\hline
\end{tabular}

As is summarized in table I, only AdHocDroid and 802.11s truly provide all the features for a MANET. As 
we mentioned our aim is to advance the state-of-the-art in MANET experimentation by providing a framework to easily test MANET network protocols. In the future, 802.11s, when it becomes adopted in chipsets and their drivers, will provide an easy establishment of a multi-hop MANET. However, as multi-hop is provided at driver level, it can be more difficult to test routing protocols than with our solution.

\section{EXPERIMENTAL RESULTS}

We performed a series of experiments to test AdHocDroid both from an end-user perspective, using standard applications (multiplayer games) from the Play Store to verify that they work unchanged in a MANET, and from a technical perspective, measuring throughput, delay and battery consumption and comparing them with values obtained in a standard infrastructured scenario with an AP. The experiments were performed using three Samsung Nexus S smartphones running Android 4.3 with CyanogenMod 10.2.1, in single (SH) and multihop (MH) configurations, as shown in Figure 3.
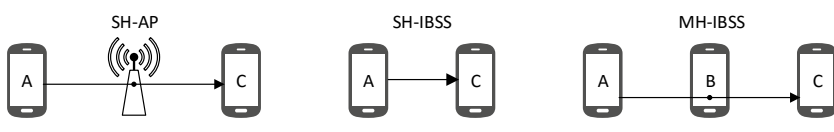

Figure 3. Test scenarios

\section{A. Standard Applications}

From an end-user perspective, we downloaded from the Play Store two different multiplayer games for the local network, 2048 BATTLE - multiplayer game ${ }^{8}$ and Spaceteam ${ }^{9}$, and tested them in single and multihop IBSS. 2048 BATTLE worked straightforward. The application asks for the last byte of the IP address of the opponent (apparently assuming a class $\mathrm{C}$ network), and connecting the two users works even in the multihop scenario. Changing between the single and multihop scenarios during the game did not disrupt it in any perceivable way.

In Spaceteam, connecting the users worked as expected in single hop, but not in multihop. This problem occurs because the game uses multicast DNS to find the opponent in the local network, but multicast traffic is not forwarded by the router (node B). After the discovery phase, the game worked normally, even if we changed from single to multihop and vice-versa during the game.

\section{B. Performance Evaluation}

We carried out experiments to validate the ad-hoc multihop functionality measuring throughput and delay, and then quantify the impact of AdHocDroid on battery discharge, an important metric for user acceptance of MANET based applications.

We measured the throughput of the different nodes in the scenarios shown in Figure 3. We generated traffic from node A to node $\mathrm{C}$ using iPerf (version 2.0.5) ${ }^{10}$, sending UDP packets at the maximum $802.11 \mathrm{a} / \mathrm{g}$ PHY rate (54 Mbits/s), thus

\footnotetext{
${ }^{8}$ https://play.google.com/store/apps/details?id=com.jtataming.BATTLE2048

${ }^{9} \mathrm{https}: / /$ play.google.com/store/apps/details? $\mathrm{id}=$ com.sleepingbeastgames. spaceteam

${ }^{10}$ https://iperf.fr/
}

overloading the channel and collecting throughput once per second. As the experiments were done while measuring battery discharge, they were done until one of the nodes had the battery depleted. We executed six of these batches.

The results are shown in Figure 4. As expected, throughput is comparable in the multihop IBSS and the infrastructured cases, and larger (more than double) in the single hop IBSS case. This is due to the absence of forwarding at an intermediate node (AP or router), implying lower medium contention.

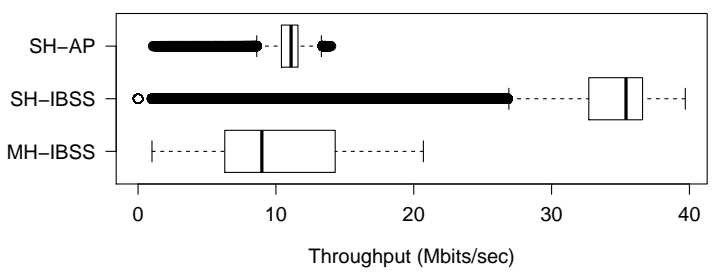

Figure 4. Throughput for infrastructure vs. IBSS single hop vs. IBSS multi-hop scenarios

To evaluate the end-to-end delay, we measured the RoundTrip Time (RTT) using ping, sending one request per second for 30 seconds. We ran six series with a one minute interval between series. Figure 5 shows the results. In IBSS mode, the RTT in multihop is about twice as large as in single hop, as expected. Through an AP, the RTT was much larger, ranging up to $400 \mathrm{~ms}$ (with the exception of some larger valued outliers). This was consistent across several models of AP (including a smartphone configured for wireless tethering but disconnected from the Internet). This large difference is probably due to the absence of power saving in IBSS mode, which is consistent with the difference in battery consumption without network traffic that we report below. Note that this does not apply to the tests with traffic, because nodes with permanent backlog do not enter the power save state. These and the previous results validate the correct functioning of the smartphone MANET.

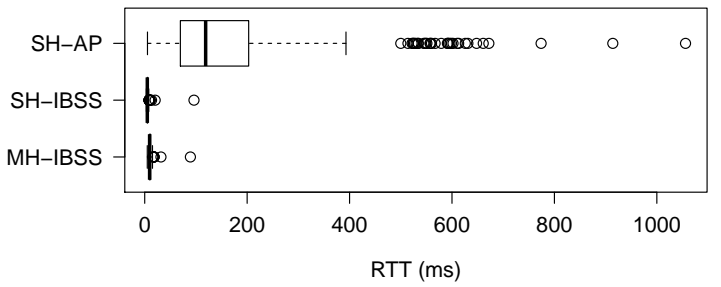

Figure 5. Ping RTT for infrastructure vs. IBSS single hop vs. IBSS multi-hop scenarios between node $\mathrm{A}$ to $\mathrm{C}$

Finally, we evaluated the impact on a smartphone's battery discharge rate of being used as MANET node. We started by measuring the battery impact of simply setting the devices to IBSS mode, without network traffic. The results are shown in Figure 6. Compared to an infrastructure scenario through an AP, there is a significant increase in battery consumption, however the OLSR daemon with the standard configurations does not further impact this consumption (note that this is a stationary scenario with only three nodes).

We also measured the discharge rate of the different nodes in the scenarios shown in Figure 3. Because the discharge rate is a function of the amount of traffic being sent/received, overloading the channel, as iPerf does, ensures that we are 


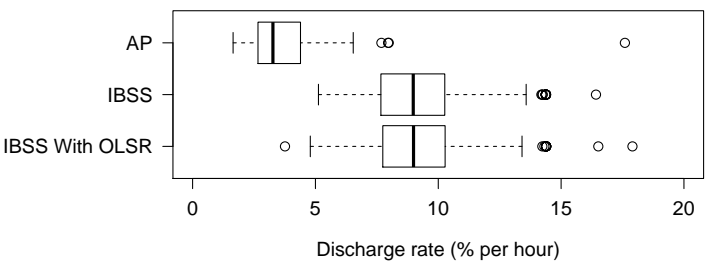

Figure 6. Discharge rate for infrastructure vs. IBSS without OLSR vs. IBSS with OLSR

observing worst-case battery discharge rates. We used the same setting as in the throughput experiments, as mentioned. In these, we additionally collected the time interval for each percent point drop in battery until the first node has the battery depleted. We plot the distribution of the discharge rate between consecutive points in Figure 7.

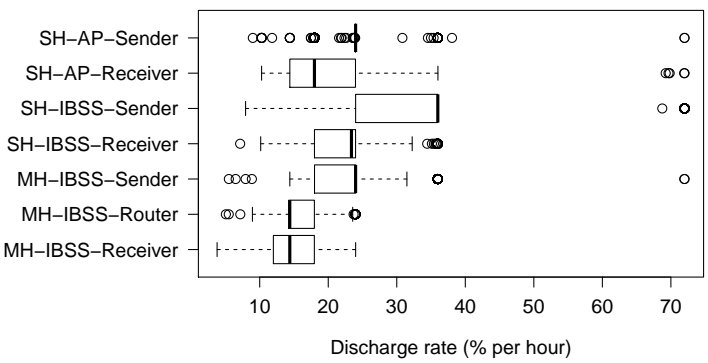

Figure 7. Discharge rate for infrastructure vs. IBSS single hop vs. IBSS multi-hop scenarios

Battery consumption in multihop IBSS is comparable to the infrastructured case for both sender and receiver. In single hop IBSS, the consumption is higher since the throughput is also higher due to the lower medium contention. Consumption at the sender is higher than at the receiver because it is trying to send at a higher rate than is actually possible. The router in the multihop IBSS scenario discharges at a similar rate as the receiver, confirming that the discharge rate is determined by the time that the network interface card is busy. We double checked this by sending at rate lower than saturation $(6 \mathrm{Mbps})$ and observing similar battery discharge rates in all nodes.

\section{CONCLUSIONS}

Motivated by a wide range of application scenarios for MANETs of smartphones proposed in the literature, we survey the existing solutions to actually enable IP-level ad-hoc networking on Android smartphones. We describe a software to enable ad-hoc networking on Android devices and shared learned lessons, so that anyone in the community can easily build a MANET test-bed with multihop capability that applications can access through the sockets API. We thus expect to contribute to moving the envisioned applications one step closer to reality.

As an example of the want of developers and others for adhoc support, we have that "Support Wi-Fi ad-hoc networking" was trouble ticket $82^{11}$ in the Android open source project, starred by 6300 people until it was classified as obsolete and closed in April 1st 2015. For comparison, the most starred ticket related to networking had been starred 9751 times and the second most starred ticket 3565 times, as of Oct 2015.

\footnotetext{
${ }^{11}$ https://code.google.com/p/android/issues/detail?id=82
}

\section{ACKNOWLEDGEMENTS}

Research funded by Project VR2Market - Ref. CMUPERI/FIA/0031/2013.

\section{REFERENCES}

[1] P. Mitra and C. Poellabauer, "Emergency response in smartphone-based Mobile Ad-Hoc Networks," in IEEE Int. Conference on Communications (ICC). IEEE, Jun. 2012, pp. 6091-6095.

[2] D. Srikrishna and R. Krishnamoorthy, "SocialMesh: Can networks of meshed smartphones ensure public access to twitter during an attack?" IEEE Communications Magazine, vol. 50, no. 6, pp. 99-105, Jun. 2012.

[3] T.-Y. Huang, C.-M. Lin, J.-R. Jiang, W. T. Ooi, M. Abdallah, and K. Boussetta, "SYMA: A Synchronous Multihop Architecture for Wireless Ad Hoc Multiplayer Games," in IEEE 17th Int. Conference on Parallel and Distributed Systems, Dec. 2011.

[4] A. Le, L. Keller, C. Fragouli, and A. Markopoulou, "MicroPlay: a networking framework for local multiplayer games," in Proc. of the 1st ACM Int. Workshop on Mobile gaming-MobileGames, New York, USA, Aug. 2012.

[5] O. Turkes, H. Scholten, and P. J. Havinga, "BLESSED with Opportunistic Beacons: A Lightweight Data Dissemination Model for Smart Mobile Ad-Hoc Networks," in Proc. of the 10th ACM MobiCom Workshop on Challenged Networks (CHANTS '15). ACM Press, Sep. 2015.

[6] A. Sathiaseelan and J. Crowcroft, "Internet on the move: Challenges and solutions," SIGCOMM CCR, vol. 43, no. 1, Jan. 2012.

[7] M. Conti and S. Giordano, "Mobile ad hoc networking: milestones, challenges, and new research directions," IEEE Communications Magazine, vol. 52, no. 1, pp. 85-96, Jan. 2014.

[8] J. Su, J. Scott, P. Hui, J. Crowcroft, E. De Lara, C. Diot, A. Goel, M. H. Lim, and E. Upton, "Haggle: Seamless networking for mobile applications," in Proc. of the 9th Int. Conference on Ubiquitous Computing. Innsbruck, Austria: Springer-Verlag, 2007.

[9] W. Kiess and M. Mauve, "A survey on real-world implementations of mobile ad-hoc networks," Ad Hoc Networks, vol. 5, no. 3, Apr. 2007.

[10] P. Mitra and C. Poellabauer, "Efficient group communications in location aware mobile ad-hoc networks," Elsevier Pervasive and Mobile Computing, vol. 8, no. 2, pp. 229-248, Apr. 2012.

[11] G. Z. Papadopoulos, K. Kritsis, A. Gallais, P. Chatzimisios, and T. Noel, "Performance evaluation methods in ad hoc and wireless sensor networks: a literature study," IEEE Communications Magazine, vol. 54, no. 1, pp. 122-128, Jan. 2016.

[12] IEEE Association, "IEEE Standard for Information technologyTelecommunications and information exchange between systems Local and metropolitan area networks-Specific requirements Part 11: Wireless LAN Medium Access Control (MAC) and Physical Layer (PHY) Specifications," IEEE Std 802.11-2012, pp. 1-2793, Mar. 2012.

[13] T. Clausen and P. Jacquet, "Optimized Link State Routing Protocol (OLSR)," IETF, RFC 3626, Oct. 2003.

[14] C. Perkins, E. Belding-Royer, and S. Das, "Ad hoc On-Demand Distance Vector (AODV) Routing," IETF, RFC 3561, Jul. 2003

[15] Wi-Fi Alliance Technical Committee P2P TG, "Wi-Fi Peer-to-Peer (P2P) Technical Specification v1.2," WiFi Alliance, Tech. Rep., 2010.

[16] W.-S. Jung, H. Ahn, and Y.-B. Ko, "Designing content-centric multi-hop networking over Wi-Fi Direct on smartphones," in IEEE WCNC, April 2014.

[17] C. Casetti, C. Chiasserini, L. C. Pelle, C. D. Valle, Y. Duan, and P. Giaccone, "Content-centric routing in Wi-Fi Direct multi-group networks," CoRR, vol. abs/1412.0880, 2014.

[18] P. Gardner-Stephen and S. Palaniswamy, "Serval mesh software-WiFi multi model management," in Proc. of the 1st Int. Conference on Wireless Technologies for Humanitarian Relief - ACWR '11, NY, USA, Dec. 2011.

[19] S. Cheshire, B. Aboba, and E. Guttman, "Dynamic Configuration of IPv4 Link-Local Addresses,” IETF, RFC 3927, May 2005.

[20] A. Aguiar, E. Soares, P. Brandão, T. Magalhães, J. M. Fernandes, and I. Oliveira, "Demo: Wireless IP Mesh on Android for Fire-fighter Monitoring," in Proc. of the 9th ACM MobiCom workshop on Challenged networks - CHANTS '14, New York, USA, Sep. 2014. 